\title{
Compliant Worm-like Robotic Mechanism with decentrally controlled Pneumatic Artificial Muscles
}

\author{
Martin Eder $^{1}$, Maximilian Karl ${ }^{1}$, Alois Knoll ${ }^{1}$ and Stefan Riesner ${ }^{2}$
}

\begin{abstract}
When human interacts with a robot, it must be guaranteed that in case of system failure no severe injury can happen. Thus either the control of the robotic device or the actuators or rather the mechanism itself have to necessarily comprise compliance. Compared to other solutions, using pneumatic artificial muscles (PAM) as actuators is an inexpensive way to end up with a mechanism that is compliant. The most challenging part here is the precision of the control, due to the highly non-linear properties of the PAM actuators [1]. This paper presents a prototypic implementation of a robotic mechanism which guarantees inherently compliant and thus human-friendly behavior. The novel idea of the shown concept is the high maneuverability due to cardan joint double axes with centrally fed actuator arrangement that allows more precise control as well as much more modularity than state of the art. A crucial challenge is the development of a smart control of the introduced mechanism, which has a labile equilibrium as a matter of principle.
\end{abstract}

\section{INTRODUCTION}

Since the interaction of human and robot always opens a debate on safety issues, it is of interest to develop systems that can reduce the dangers associated with such an interrelationship or even remove it. A core topic here is compliance, which means that the stiffness of the robot is reduced in different ways. Only if it is guaranteed that in case of collisions the risk of injury is minimal, a robot is safe enough to operate together with humans. There are quite a number of robotic systems offering compliant behavior using either adequate control strategies [2], [3], [4] to make the system soft or utilizing compliant parts of the structure and actuators, respectively (passive stiffness). Provided that low cost aspects, low energy and light-weight issues as well as intrinsic softness of the actuators are prerequisites, PAM actuators are appropriate means for driving such inherently compliant mechanisms. Compared to commercially available electric drives, PAM are low priced and very energy-efficient. In the following, some relevant state of the art mechanisms are highlighted.

At the Artificial Intelligence Laboratory of Stanford University, Stanford, California, a human-friendly robot was developed, including both PAM and electric motors to drive the joints [5], [6]. The performance of this setup with respect to the high frequency control due to fast electric motors is very high, but the drawback is the necessity that additional

\footnotetext{
${ }^{1}$ M. Eder, Maximilian Karl and Alois Knoll are with Faculty of Informatics, Robotics and Embedded Systems, Technische Universität München, D-85748 Garching bei München, Germany ederma, karlma and knoll at in.tum. de

${ }^{2} \mathrm{~S}$. Riesner is with Robotics Technology Leaders GmbH, D-81249 München, Germany s.riesner at rtleaders.com
}

electric drive units have to be integrated, which increases cost, weight and development effort of the system.

A robot with two degrees of freedom (DOF) driven by four artificial muscles is presented in [1], developed by the Institute of Automation and Systems Engineering TU Ilmenau, Germany, in cooperation with Festo AG \& Co. KG. It consists of a flat articulated system that can just move in a plane. Hence, motion capability is strongly restricted.

Another very famous mechanism is the Bionic Handling Assistant (BHA) built by Festo AG \& Co. KG, which is highly flexible because of the parallel arrangement of several bendable segments based on 3D printed plastics [7]. The control devices for this BHA are completely centralized within a valve cluster underneath the basement, containing quite large proportional directional control valves, type Festo VPWP ${ }^{1}$.

In the following paper, the prototypic implementation and test of a robotic system is described which aims towards the improvement of current control strategies of inherently compliant mechanisms based on PAM. Main difference compared to state of the art systems is that the mechanism here is based on locally arranged control and supply units within segments. These decentral control units in combination with a central feed guarantee independent control of each of the mechanism's segments. The central feed is realized by means of a central feed line that supplies locally mounted valves with pressurized air, each of them fixed very close to the muscle [8]. Due to the very short distance between the feed line, the valves and the muscles, minimal dead volume results which allows more precise controllability. By reducing the dead volume, oscillations during filling or exhausting decrease. Particular attention is paid to high maneuverability, therefore each subsystem of the mechanism can rotate in two DOF, utilizing cardan joints that generate an actually inherently labile equilibrium composition, which makes a position control even more challenging than what so far is known within state of the art. Every axis of the cardan joints is equipped with a rotational encoder such that a detection of all rotations is possible. The system can be extended modularly to a various number of segments, thus making the mechanism arbitrary in terms of length.

The specific contribution within the work presented in this paper is a concept of a novel compliant PAM mechanism offering high maneuverability due to cardan joint double axes together with a centrally fed actuator setup, which results in more precise controllability and enhanced modularity

\footnotetext{
${ }^{1}$ each with the size of $132 \mathrm{~mm} \times 60 \mathrm{~mm} \times 74 \mathrm{~mm}$
} 
compared to state of the art constructions.

Within the scope of this paper, section II illustrates the design of our robotic system in detail, section III describes specifics about the control, whereas section IV shows the results of our approach. In a final step, conclusions and future works within our worm-like mechanism project are summarized in section V.

\section{Mechanism Design}

The focus of attention within the mechanism design is a special development of independently controllable segments all with locally fixed actuators and actuator control units. Each segment comprises the following elements, as shown in Fig. 1:

An aluminum light-weight base plate is used to carry an aluminum rod with a plastic valve cluster holder for eight valves controlling four artificial muscles that are arranged in antagonistic placement. The weight of the muscles used is $60 \mathrm{~g}$ for DMSP-10-160N-RM-CM type having a maximum drag force of 630N [9], [10]. The plastic holder as well as the manifolds for all the fittings, the pressure sensor and the exhaust silencer, are based on a selective laser sintering rapid prototyping part made of PA2200, which is biocompatible under the terms of DIN EN ISO 10993-1. The muscles (maximum 6bar) positioned opposite to each other generate two rotational DOF about a central cardan joint that is fixed to the rod. Each muscle is connected to two proportional valves $^{2}$, one for filling, one for venting. Former simple binary on-off miniature valves ${ }^{3}$ turned out to be too slow with much less performance in terms of oscillation effects than the new proportional valves. Sensorial feedback information from the muscles is conducted via small pressure sensors ${ }^{4}$ with $\pm 0.3 \%$ repeatability. The information about the two tilting angles of the cardan joint is measured with two magnetic absolute encoders with 12 bit resolution ${ }^{5}$, i.e. less than $0.1^{\circ}$, used with diametric magnets attached to the rotating axes. A PAM distance piece prevents the muscles to collide against any other component, in particular against these rotary encoders. On the second side of the cardan joint another aluminum plate is attached which is the anchor part for the following segment. The real setup of this $1^{\text {st }}$ segment of the mechanism is depicted in Fig. 2.

The arrangement of several segments (one in our current setup, two more are already in preparation) results in a serial kinematics with four parallel actuators per segment. The setup described is shown in Fig. 3, including these two additional segments.

A technical specification of the complete assembly of the worm-like mechanism according to Fig. 3 is highlighted in Tab. I.

${ }^{2}$ Parker MD Pro miniature proportional valve, non-thermally compensated, 2-way normally closed, orifice $1.27 \mathrm{~mm}$

${ }^{3}$ by FAS, type Chipsol

${ }^{4}$ SMC PSE-510-M5-Q, analog

5 type AS5145H-HSST SSOP16 LF by ams

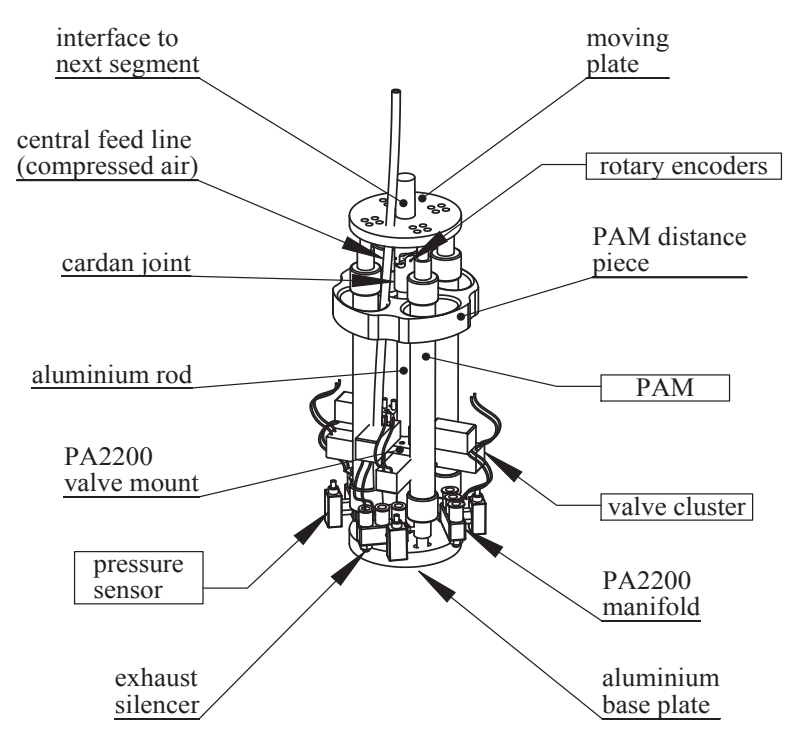

Fig. 1. $1^{\text {st }}$ segment of worm-like robotic mechanism

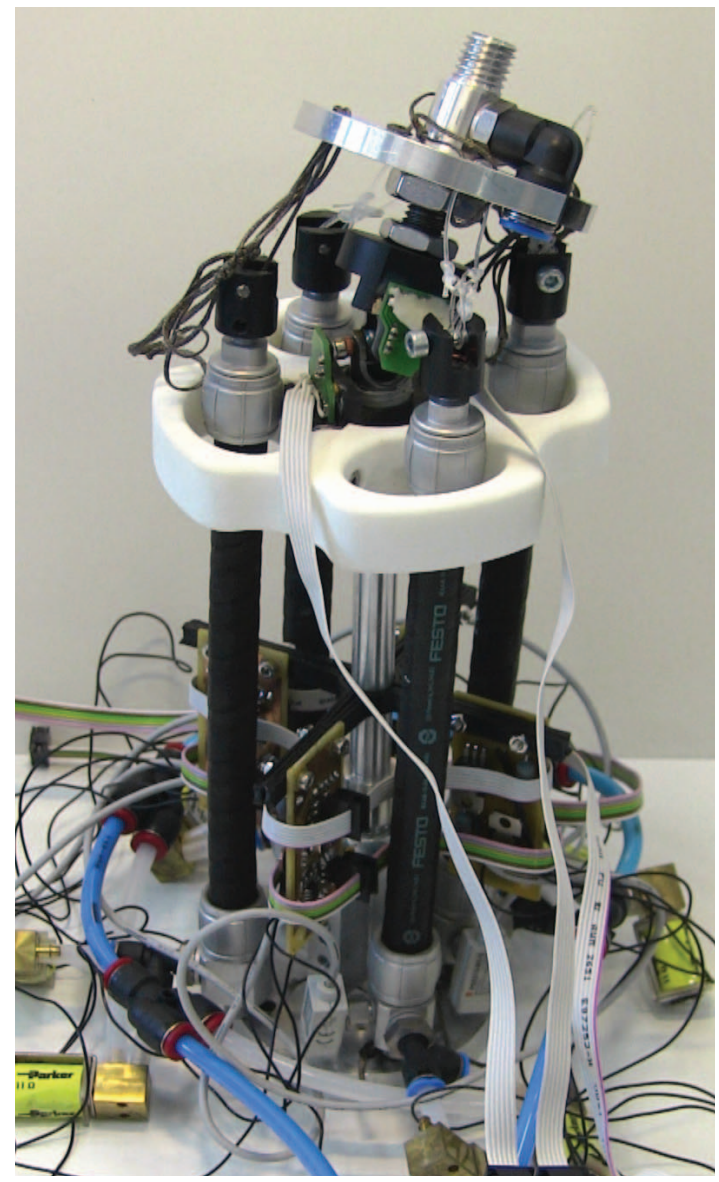

Fig. 2. Real setup: $1^{\text {st }}$ segment of worm-like robotic mechanism 


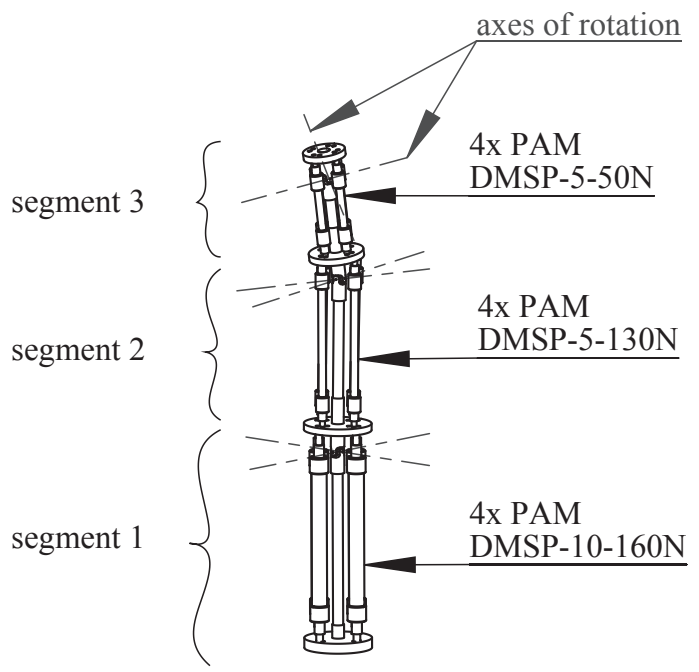

Fig. 3. 3 segments of worm-like robotic mechanism

TABLE I

SPECIFICATION

\begin{tabular}{lr}
\hline feature & value \\
\hline number of PAM & 12 \\
PAM type & Festo DMSP \\
number of valves & 24 \\
voltage supply & $5 / 9 \mathrm{VDC}$ \\
air supply (maximum) & $6 \mathrm{bar}$ \\
length of segment 1 & $260 \mathrm{~mm}$ \\
length of extra segment 2 & $200 \mathrm{~mm}$ \\
length of extra segment 3 & $140 \mathrm{~mm}$ \\
overall length & $600 \mathrm{~mm}$ \\
maximum diameter & $80 \mathrm{~mm}$ \\
maximum joint angles of segment 1 & $\approx \pm 20^{\circ}$ \\
maximum joint angles of extra segment 2 & $\approx \pm 20^{\circ}$ \\
maximum joint angles of extra segment 3 & $\approx \pm 20^{\circ}$ \\
frame material & aluminum \\
manifold material & PA2200 \\
weight of segment 1 & $\approx 600 \mathrm{~g}$ \\
overall weight (including segments 2 and 3) & $\approx 1600 \mathrm{~g}$ \\
\hline
\end{tabular}

\section{Control Strategy}

Detailed model-based controlling of the aforementioned mechanism is hardly feasible with reasonable expense. Due to highly non-linear system behavior, not all parameters can be calculated or estimated rationally, e.g. internal friction or damping of the muscle rubber material, temperature influence etc. The main reasons for non-linearity are compressibility of the fluid and flow within the valves [11], [12]. Thus our approach is based on a rather simple PID force controller that deals with precisely pre-calibrated PAM. In this respect too complex algorithms that also might result in problems in terms of real-time capability are avoided.

\section{A. Muscle characteristics}

One of the most important steps for the control of PAM is calibration of the muscles or rather gaining their characteristics [13], [14]. To simplify the modeling, the muscle behavior is examined under quasi-static state. The characteristics that are measured create the correlation between muscle pressure, the external adjustable calibration force, with which the mechanism is loaded, and the resulting joint angle (cf. Fig. 4). This calibration indirectly also implies the shortening of the muscle. With the quasi-static pulling tests by means of defined balance weight and lever arm, acting as a determined calibration force, the characteristics are obtained. In order to enhance the performance of the control later on, the measured characteristics for each PAM are transformed into low complex but accurate functions that can be easily computed by microprocessors. In a first step, for each muscle the muscle model introduced by Hildebrandt [1] is used for identification of these functions, considering the PAM as one way cylinders moving against a spring, where $p$ is the pressure, $f$ represents the muscle force and $l$ denotes the current length of the muscle which corresponds to the joint angle according to kinematics:

$$
p(f, l)=f_{1}(l) f+f_{2}(l)
$$

In a second step, the characteristics are fitted via performing an evolutionary symbolic regression approach utilizing software tool Eureqa ${ }^{6}$. In this step, the function shape is based on the muscle model mentioned in the previous step. In Fig. 4 the characteristics for one PAM are illustrated. Whereas forces are denoted in $N$ and angles in ${ }^{\circ}$, the pressure is shown in bar. The results for the low complex functions fitted for all four muscles $(I-I V)$ of our $1^{\text {st }}$ mechanism segment are as follows:

$$
\begin{aligned}
p_{I}(f, l)= & 9.51 f l+1329.57 f l^{4}-20.97 f l^{2}-2932.46 f l^{5} \\
- & 151.04 l^{3}+65.62 l^{2} \\
p_{I I}(f, l) & =3.79 f+232.76 f l^{3}-390.07 f l^{4} \\
& -1489.71 f l^{5}+2095.55 f l^{6}-73.83 l^{4}+2.00 \\
p_{I I I}(f, l)= & \frac{f}{0.12+0.23 l}-\frac{l-0.38}{0.11+0.04 l-0.43 l^{2}-0.22 l^{3}} \\
p_{I V}(f, l) & =7.18 f-215.57 l^{5}+2.81+1.62 l^{2}-4.75 l
\end{aligned}
$$

\section{B. Pressure control}

Whereas in a former stage of development simple on-off binary valves (FAS Chipsol) were used, now the setup is equipped with small proportional valves, Parker MD Pro. The old valves suffered one major drawback that depending on the input pressure at the start only PWM duty cycles within the range of $10-75 \%$ were practicable. With the MD Pro valves a pressure control for each antagonistic pair of PAM is feasible which behaves very sensitively. For each muscle, a pressure controller is utilized. The analog signals of the pressure sensor are conditioned with an ADC and interpreted

\footnotetext{
${ }^{6}$ see creativemachines.cornell.edu/eureqa
} 


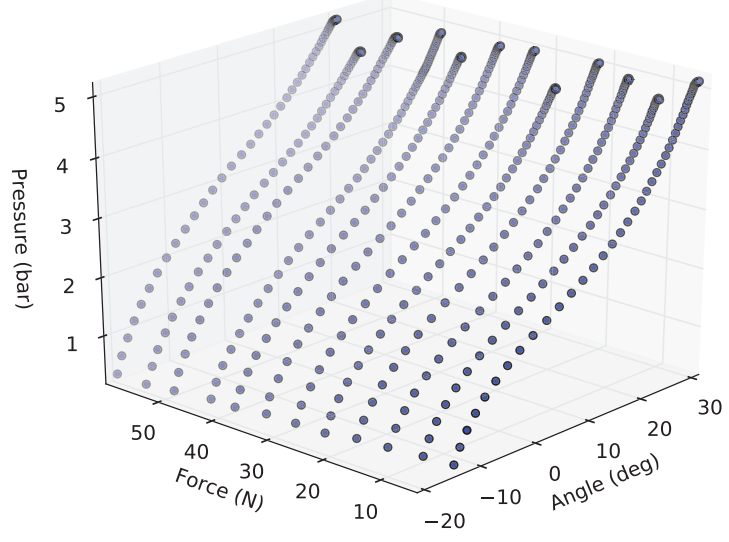

Fig. 4. Muscle characteristics

by a local microcontroller that feeds the current controller for both inlet and outlet valve. Since the PAM can only generate tensile forces, prestressing of the PAM is necessary such that the pair of PAM can move in two directions creating a rotational movement about the cardan joint axis. Due to this, half of the shortening length of the PAM, i.e. $12.5 \%$, is used for prestressing, so both further shortening and extension by the same value is possible. The schematics of the pressure controller is depicted in Fig. 5. Details about its integration into a superordinate angle control and especially into a global control hardware architecture are given in III-C and III-D.

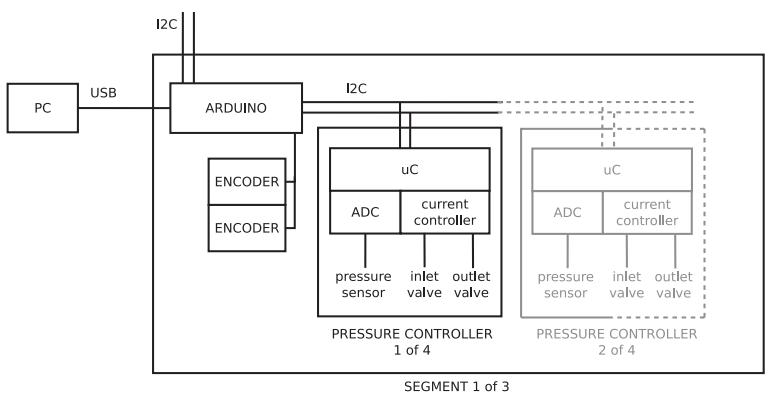

Fig. 5. Pressure controller with decentral hardware architecture

\section{Angle control}

Superior to the interior pressure controller based on input data from the pressure sensors attached to the PAM, there is an outer angle controller utilizing the data from the rotary encoders, such that a cascaded control loop is performed, which is responsible for two antagonistic pairs of PAM. A trajectory generator unit, performed by an overall control PC, generates the input commands for angular position, which a PID controller processes that in turn is responsible for one pair of PAM each. These values are added to a force controlling stage $^{7}$ solving the muscle forces, that also considers stiffness

\footnotetext{
7 internally forces are treated as moments of forces since the forces always have a lever arm with respect to the cardan joint axes
}

forces and optionally additional external forces. Since the two pairs of PAM are kinematically dependent of each other due to the overslung mechanical arrangement around the cardan joint (if one pair is moving, the length of the $2^{\text {nd }}$ pair automatically changes), the forces of all four muscles have to be considered simultaneously. Therefore a system of linear equations is formed that correlates the forces for four muscles considering the mechanism's geometry or rather the desired angle such that an equilibrium state results. The muscle force solver (see Fig. 6) thus computes these equations, adding additional loads or stiffness loads if needed. By means of the desired angles and the calculated forces for all four muscles of one segment, the output pressures are computed while taking the muscle characteristics into account. The resulting output pressures for both muscle pairs make the robotic mechanism move. The actual values of the angles measured by the rotary encoders are fed back to the overall angle control loop. Since cardan joints are utilized that move an overslung plate, a mechanically unstable equilibration results, which has to be handled with a sensitive control, for there is a strong mutual interference of both rotational motions or two angles, respectively. The stiffness forces can be set arbitrarily depending on how stiff the behavior of the muscle pairs should be. For both PAM of one pair the same force value is added to the force control part. In case that there are further external forces, such as forces from supplementary loads, these forces can also be added to this part of the control loop. With every change of the output pressure and the angle, respectively, there will be a new equilibrium state of the entire system, which means that the forces of the PAM are equal to the external forces the robotic mechanism is loaded with. The overall correlation of the angle control for one segment is shown in Fig. 6.

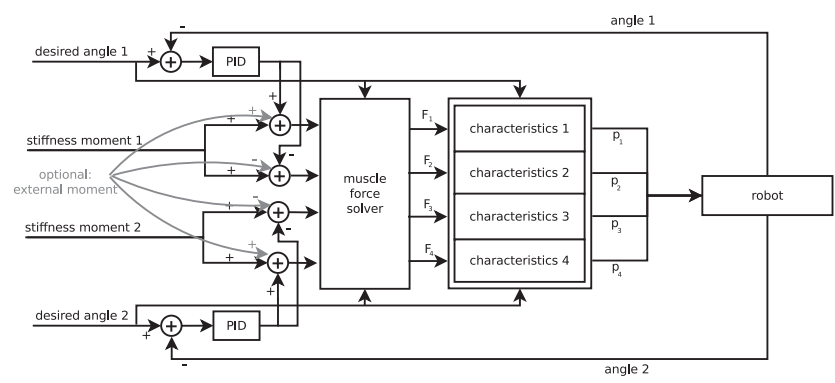

Fig. 6. Angle control

\section{Control hardware architecture}

Each of the segments of the worm-like robot is equipped with an Arduino Nano 3.0 board using an ATmega 328 microcontroller, providing an $i^{2} C$ bus by means of which the segments are connected (two additional segments are prepared to be attached to current $1^{\text {st }}$ segment). Every Arduino board is mounted on an adapter board which offers input connections for two rotary encoders of the cardan joint as well as the $i^{2} C$ bus interface. Additionally, all of the superior Arduino boards are again connected with four 
more inferior pressure controller boards. In each case one pressure sensor and two proportional valves (one inlet valve, one outlet valve) are linked to a pressure controller board. In turn, these slave boards are connected via $i^{2} C$ bus. The $1^{\text {st }}$ Arduino Nano board is connected to a superordinate control and GUI PC using a USB bus connection. Thus the architecture of our control is fully decentrally arranged which means that the setup is completely modular. An overview of this decentral control hardware architecture is visualized in Fig. 5, incorporating the aforementioned pressure controller (cf. III-B).

\section{EXPERIMENTAL RESULTS}

To prove the high performance of the system that was developed, various analyses were employed, in particular to demonstrate the effectiveness of the decentral control concept and the use of a double axes system in the form of a cardan joint for motivity reasons.

After calibration of one pair of PAM of the $1^{\text {st }}$ segment, moving experiments were conducted without additional load. In order to intuitively estimate the performance, a rotary sine movement of the mechanism with PID control was chosen. In Fig. 7(a) the results of this test comparing actual angles (colored in blue) and desired angles within a given trajectory (colored in grey) are illustrated (subfigure on the left). The average angle error during the sine motion is about $0.14^{\circ}$ (subfigure on the right).

Having calibrated all four PAM of the $1^{\text {st }}$ segment, a similar movement was conducted in which both DOF of the cardan joint are controlled in a sinusoidal manner. Again, for this motion actual angles (colored in blue and red) are compared to desired angles (colored in grey). Fig. 7(b) resumes the results of these tests. Here, the average angle error during the sinusoidal motion is in the range of $\approx 0.36^{\circ}$ for the $1^{\text {st }}$ pair (blue) and $\approx 0.49^{\circ}$ for the $2^{\text {nd }}$ pair (red).

Several more experiments were conducted applying different loads to the mechanism, having mounted the setup in horizontal configuration employing constant torques by adding weights at defined lever arms such that mainly the $1^{\text {st }}$ pair of PAM (colored in blue) is loaded whereas the $2^{\text {nd }}$ pair is rather unloaded (colored in red) . In Fig. 7(c) an additional load of $0.71 \mathrm{Nm}$ is used, in Fig. 7(d) $2.08 \mathrm{Nm}$ are loaded, in Fig. 7(e) even $2.51 \mathrm{Nm}$ are set. It can be seen that the (mainly) loaded muscles only marginally change their precision in motion, the (almost) unloaded ones even improve their performance. If the achievements from the aforementioned coupled movements are compared to state of the art mechanisms, the error angles are as good as for instance in the setup of [1]. This is a very pleasant result, because in the setting of [1] the motions of the two DOF are completely decoupled from each other. Since in our case there is a direct kinematic connection of all four PAM of one segment and because of the existence of a lever movement through the cardan joint, respectively, there is a mutual influence of the two DOF, i.e. if a $1^{\text {st }}$ pair of PAM is moving, the $2^{\text {nd }}$ pair of PAM is slightly affected in its motion. Thus the performance quality of two simultaneously moved pairs of PAM is a little worse than of one pair only. The difference between the two pairs of PAM is due to the fact that each PAM has marginally distinct material properties and additionally the calibration of each muscle is conducted only with a limited amount of calibration steps and resolution, respectively. In spite of the already mentioned labile equilibrium of the overslung arrangement of moving plate, cardan joint and stationary part of the segment, it is proven that surprisingly smooth motions are feasible. The smoothness is even given during the motion over the dead centre, i.e. when the moving plate rotates from one side to the other side crossing the horizontal pose. Furthermore, the results during movements with additional loads fortunately show performance as good as in unloaded state.

\section{CONCLUSION}

The presented mechanism has numerous advantages compared to mechanisms of best available technology utilizing PAM. Most decisive point is the precision of the muscle control due to locally arranged valves with extremely short pipes to the actuators as well as the use of cardan joints with two DOF making the mechanism highly maneuverable. The advantage of precision because of the valves that are directly connected to the PAM increases the more segments are integrated in the mechanism. In case of mechanisms with many serially arranged segments, alternatively arranged feed lines from centrally allocated valves, e.g. mounted on a valve cluster basement, would be very long and consequently the dead volume in between causes control drawbacks such as slow reaction time and additional oscillations. Moreover, the local fixing of actuator control technology such as valves and PCB reduces the total outer diameter especially for mechanisms with a large number of segments. Else there would be lots of cables and pneumatic tubes necessary which are lead through the predecessor segments [15]. As a result of the implementation of precise cardan joints, each segment has two DOF and the mechanism can move in various directions smoothly, in particular being able to rotate in two DOF about a single pivot point simultaneously, which makes the presented mechanism unique in contrast to current state of the art. Since flexible pneumatic actuators are utilized, soft motions are feasible such that a collaboration mode with humans is possible. In this respect, the system inherently meets safety requirements. Because of the decentral design of all components, the whole setup is modular and scalable. Thus attaching more segments to an existing setup is feasible without hardware adaption problems.

Although the feasibility of our decentral control approach and the advantage of using two DOF cardan joints in terms of smooth and capable maneuverability about one pivot point is already proven, the setup shall still be extended to carve out the benefits for real applications. An aim of forthcoming development is the expansion to fields of applications that deal with the interaction between humans and machines. Hence, for the near future, the setup will be enlarged to three segments, each with four PAM, thus 12 PAM in total, where the decentral control architecture together with the locally arranged air supply units is implemented again. In the distant 


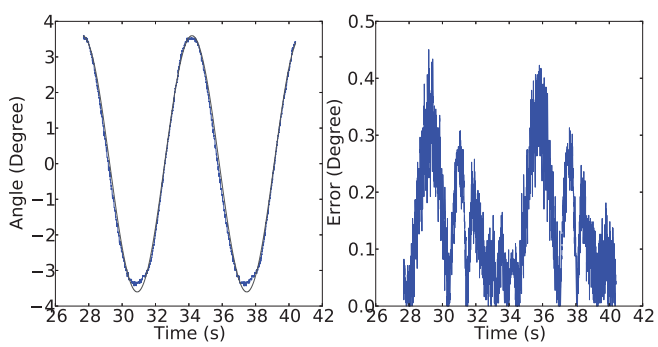

(a) Sine motion of one PAM pair

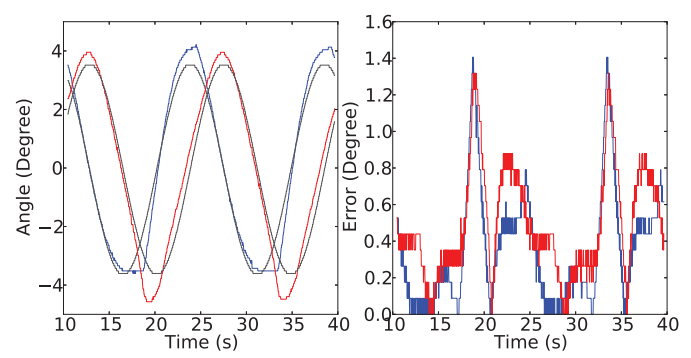

(b) Sinusoidal motion of two PAM pairs

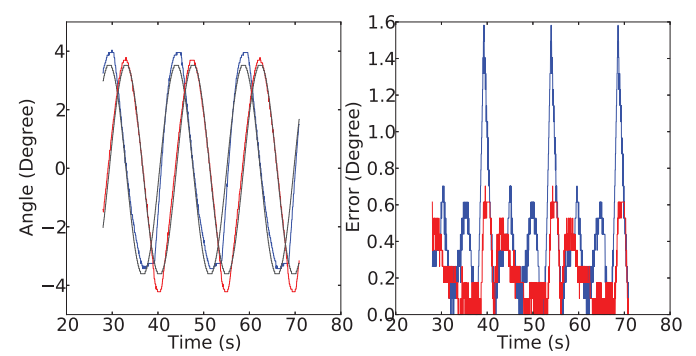

(c) Sinusoidal motion of two PAM pairs with $0.71 \mathrm{Nm}$ load

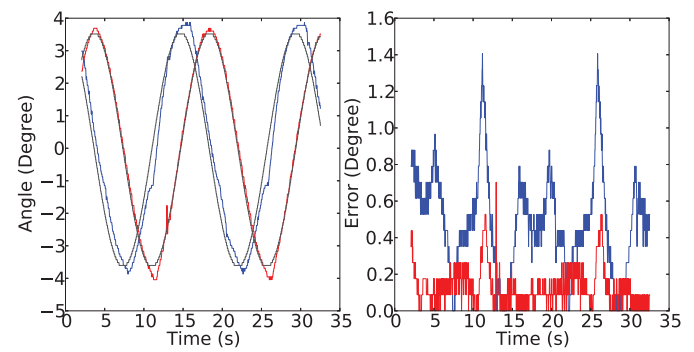

(d) Sinusoidal motion of two PAM pairs with $2.08 \mathrm{Nm}$ load

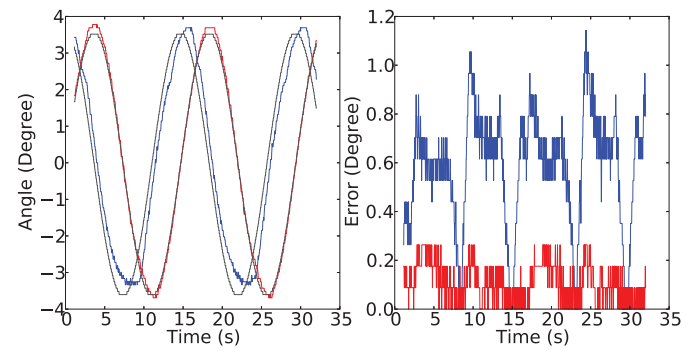

(e) Sinusoidal motion of two PAM pairs with $2.51 \mathrm{Nm}$ load

Fig. 7. Results of movement experiments: movements in grey (desired angles), blue (actual angles of $1^{\text {st }}$ PAM pair) and red (actual angles of $2^{\text {nd }}$ PAM pair) on the left subfig., corresponding angle errors on the right subfig. future, even more than three segments will be integrated in order to prove the concept for large scale systems, resulting in a mechanism that can move in worm-like manner, either on the ground or free-standing.

\section{ACKNOWLEDGMENT}

The authors gratefully acknowledge the contribution of Robotics Technology Leaders GmbH München for technical support, Festo AG and Co. KG Esslingen for the donation of PAM and ams AG for the donation of rotary encoders.

\section{REFERENCES}

[1] A. Hildebrandt, O. Sawodny et al. "Cascaded control concept of a robot with two degrees of freedom driven by four artificial pneumatic muscle actuators". In Proceedings of the 2005 American Control Conference, June 8-10, 2005, Portland, USA, pages 680-685

[2] F. Lange. "Verfahren zur Kraftregelung". DE patent application DE 102009040194 A1, September 7, 2009.

[3] R. Bischoff, J. Kurth, G. Schreiber et al. "The KUKA-DLR Lightweight Robot arm - a new reference platform for robotics research and manufacturing". In: Joint 41th International Symposium on Robotics and 6th German Conference on Robotics, 2010. KUKA Roboter GmbH, Germany; Deutsches Zentrum für Luft- und Raumfahrt, Germany.

[4] K. Kassow, E. H. Oestergaard, K. Stoy. "Programmable Robot and User Interface". International patent application WO 2007/099511 A2, March 2, 2007.

[5] S. Dongjun, I. Sardellitti, O. Khatibb. "A Hybrid Actuation Approach for Human-Friendly Robot Design". In: Proceedings of the IEEE International Conference on Robotics and Automation, pages 17471752, Passadena, USA, May 2008

[6] S. Dongjun, F. Seitz, O. Khatik, and M.R. Cutkosky. "Analysis of Torque Capacities in Hybrid Actuation for Human-Friendly Robot Design". In Proceedings of the IEEE International Conference on Robotics and Automation, Anchorage, USA, May 2010

[7] Festo AG \& Co. KG. "Bionic Handling Assistant". Info-brochure. Festo, Esslingen, Germany, 2010

[8] A. Knoll. "Worm-like mechanism". U.S. Patent US 8,201,473 B2, June 19, 2012.

[9] Festo AG \& Co. KG (publisher). "Fluidic Muscle DMSP/MAS". Info 501. Festo AG \& Co. KG, 2008

[10] S. Hesse. "Der Fluidic Muscle in der Anwendung. 150 Praxisbeispiele mit dem Pneumatischen Muskel". Blue Digest on Automation. Festo, 2003

[11] A. Hildebrandt. "Regelung und Auslegung servopneumatischer Aktuatorsysteme". PhD thesis, D93 Shaker Verlag Aachen, 2009

[12] F. Daerden. "Conception and Realization of Pleated Pneumatic Artificial Muscles and their Use as Compliant Actuation Elements". $\mathrm{PhD}$ thesis, Vrije Universiteit Brussel, July 1999

[13] T. Kerscher, J. Albiez et al. "Evaluation of the Dynamic Model of Fluidic Muscles using Quick-Release". In: The First IEEE/RAS-EMBS International Conference on Biomedical Robotics and Biomechatronics, 2006, pages 637-642, Pisa, Italy, ISBN 1-4244-0040-6

[14] C.-P. Chou, B. Hannaford. "Measurement and Modeling of McKibben Pneumatic Artificial Muscles". In: IEEE transactions on robotics and automation 12 (1996), February, Nr. 1, S. 90-102

[15] S. Schulz. "Wurmförmiger Arbeitsmechanismus". DE patent application DE 19833340 A1, July 24, 1998. 